\title{
Obtención de péptidos antioxidantes de glicinina de soja
}

\author{
Medrano, A. ${ }^{(1) *}$, del Castillo, M. D. ${ }^{(2)}$ \\ (1) Departamento de Ciencia y Tecnología de Alimentos. Facultad de Química, Universidad de la República, Uruguay. \\ (2) Instituto de Investigación en Ciencias de la Alimentación. CSIC, España.
}

Contacto: medranoalejandra@gmail.com

Recibido: 30/06/2011 - Aprobado: 7/11/2011

\begin{abstract}
$\underline{\text { Resumen }}$
El objetivo de este trabajo fue evaluar la potencialidad de dos preparados enzimáticos, proteasas de Streptomyces griseus (PSG) y de Aspergillus oryzae (Flavourzyme), en la obtención de péptidos antioxidantes de glicinina de soja. La reacción de hidrólisis se realizó bajo condiciones controladas de pH, temperatura y tiempo. El grado de hidrólisis (GH\%) se estimó mediante determinación de grupos amino libres. Los hidrolizados obtenidos se caracterizaron por SDS-PAGE y análisis de sus propiedades antioxidantes por ABTS, ORAC y oxidación de desoxirribosa. Los compuestos fenólicos se determinaron por reacción con Folin-Ciocalteu. El GH\% dependió del tiempo de reacción y de la naturaleza del preparado enzimático, con Flavourzyme más activa que PSG $(p \leq 0.05)$. Se observó una correlación directa entre GH\% de Flavourzyme y la acción frente a radicales ABTS e hidroxilos $(p \leq 0.05)$ de sus hidrolizados. Los hidrolizados de PSG resultaron significativamente más efectivos $(p \leq 0.05)$ frente a radicales alquilperoxílicos que los de Flavourzyme. No se detectaron diferencias significativas $(p>0,05)$ en las concentraciones de compuestos fenólicos de las muestras, por lo que las diferencias en capacidad antioxidante de los hidrolizados proteicos se pueden asociar a su composición peptídica. Las investigaciones de este equipo remarcan el interés del análisis de fenoles en hidrolizados proteicos con propiedades antioxidantes.

Palabras clave: glicinina de soja, péptidos antioxidantes, hidrólisis enzimática, proteasas de Streptomyces griseus (PSG), proteasas de Aspergillus oryzae (Flavourzyme).
\end{abstract}

\begin{abstract}
$\underline{\text { Abstract }}$
The aim of this study was to evaluate the potential of two enzymatic preparations, proteases from either Streptomyces-griseus (PSG) or Aspergillus oryzae (Flavourzyme), for the obtaining of antioxidant peptides from soybean glycinin. The hydrolysis was performed under controlled conditions of $\mathrm{pH}$, temperature and time. The degree of hydrolysis (GH\%) was estimated by analysis of free amino groups. Hydrolysates were characterized by SDS-PAGE and analysis of their antioxidant properties by ABTS, ORAC and oxidation of deoxyribose assays. Phenols were determined by Folin-Ciocalteu method. DH\% depended on the hydrolysis time and the nature of the enzymes being Flavourzyme more effective than PSG $(p \leq 0.05)$. A direct correlation between the DH\% of Flavourzyme and the activity of its hydrolysates against ABTS and hydroxyl radicals $(p \leq 0.05)$ was found. PSG's hydrolysates resulted significantly $(p \leq 0.05)$ more active against alkyl peroxyl radicals than those of Flavourzyme. The concentration of phenols was not significant different $(p>0.05)$ among the samples. Differences in antioxidant capacity among the samples may be associated to their peptides. Our research underlines the interest for the analysis of phenol in protein hydrolysates with antioxidant properties.

Keywords: soybean glycinin, antioxidant peptides, enzymatic hydrolysis, proteases from Streptomyces griseus (PSG), proteases from Aspergillus oryzae (Flavourzyme).
\end{abstract}

\section{Introducción}

Soja (Glycine max) es un cultivo oriundo de Asia oriental. La proteína de soja puede ser fraccionada en cuatro grupos principales sobre la base de la velocidad de sedimentación (2S, 7S, 11S y 15S). Las proteínas de soja más abundantes $(70 \%)$ son las globulinas, glicinina (11S) y conglicinina (7S). La glicinina es una proteína hexamérica con un peso molecular (PM) de alrededor de $350 \mathrm{kDa}$, que consta de cinco subunidades (A1aB2, A1bB1b, A2B1a, A3B4 y A5A4B3), cada sub-unidad compuesta de un polipéptido ácido de 37 a $42 \mathrm{kDa}$ y uno básico de $20 \mathrm{kDa}$ unidos por un puente disulfuro (Staswick et al., 1984; Nielsen, 1985).

El uso de proteína de soja como ingrediente funcional en los alimentos continúa generando aceptación debido a los beneficios para la salud y a su bajo costo, al ser un subproducto de la industria del aceite y el biodisel. Estudios sugieren que las proteínas de los alimentos, como proteínas de la leche (Hogan et al., 2009; Liu et al., 2007; Gómez et al., 2008), proteína de soja (Moure et al., 2006; Chen et al., 1995) y proteínas de huevo (Sakanaka, 2004, 2006) han presentado propiedades antioxidantes, atribuidas a ciertas secuencias específicas (péptidos) de las proteínas. Un antioxidante se define como "cualquier sustancia que, estando presente en baja concentración en comparación con las de un sustrato oxidable, retrasa o previene significativamente la oxidación de ese sustrato". La actividad antioxidante es especialmente importante, ya que la oxidación es una reacción inevitable en todos los organismos vivos (Halliwell, 1996a, 1996b). Los radicales libres y especies reactivas del oxígeno (ROS), nitrógeno y las especies de cloro contribuyen al desarrollo de varias enfermedades relacionadas con la edad, mediante la inducción de estrés oxidativo y el daño oxidativo. Debido a las consecuencias negativas de los procesos oxidativos, su inhibición es importante, por lo que la búsqueda de compuestos con actividad antioxidante es un área de intensa investigación.

El objetivo de este trabajo fue evaluar la potencialidad de los 
preparados enzimáticos PSG y Flavourzyme en la obtención de péptidos con propiedades antioxidantes a partir de un aislado de glicinina de soja.

\section{Materiales y Métodos}

La obtención del aislado de glicinina se realizó a partir de porotos de soja desgrasados con hexano durante 6 horas, según el procedimiento estándar AOAC 920.85 (AOAC, 1990) y de acuerdo al procedimiento propuesto por Nagano et al. (1992), seguido por la dispersión del precipitado en medio alcalino $(\mathrm{pH} 8,0)$ y posterior liofilización. El contenido de proteína se determinó por el método de Lowry et al. (1951) y Kjeldahl (AOAC, 1975). El estudio térmico de la proteína se realizó con calorímetro diferencial de barrido Shimadzu DSC 50. El software utilizado fue el thermal analysis system TA50 WSI. Todas las sustancias químicas utilizadas fueron de grado analítico.

\section{Preparación de hidrolizados de glicinina}

La glicinina se hidrolizó con PSG y Flavourzyme, ambas Sigma Chemical Co. (St. Louis, USA). En primer lugar, la glicinina se disolvió en agua destilada $5 \%(\mathrm{w} / \mathrm{v})$, la suspensión se ajustó a $50^{\circ} \mathrm{C}$ y pH 8.0. La reacción se inició mediante la adición de las enzimas con una relación molar enzima:sustrato 1:50 y continuó durante 4 horas manteniendo el pH constante con la adición de NaOH.0,1 M. Después de la reacción, la mezcla se calienta a $85^{\circ} \mathrm{C}$ durante $10 \mathrm{~min}$ para inactivar la enzima y se centrifuga a $3500 \mathrm{~g}$ durante $10 \mathrm{~min}$ a $4{ }^{\circ} \mathrm{C}$. El sobrenadante fue liofilizado y almacenado a $-20^{\circ} \mathrm{C}$.

\section{Caracterización de los hidrolizados}

El grado de hidrólisis $(\mathrm{GH} \%)$ se estableció mediante la determinación de los grupos amino libre por el método del oftaldehido (OPA), de acuerdo a lo descrito por Goodno (1981). Las determinaciones fueron realizadas por triplicado utilizando una curva patrón de N- $\alpha$-Acetil-L-Lisina L-leucina y medida de absorbancia a $420 \mathrm{~nm}$.

$$
\mathrm{GH}=\frac{\text { Número de enlaces peptídicos hidrolizados }}{\text { Número total de enlaces peptídicos }} \times 100
$$

La determinación de fenoles totales se realizó mediante el método de Folin-Ciocalteu, utilizando ácido gálico como patrón (Kosar et al., 2005).

El grado de pureza de la glicinina y la reacción de hidrólisis se siguió utilizando geles comerciales de Bis-Tris NuPAGE 12\% (Invitrogen, Carlsbad, CA), empleando tampón de separación NuPAGE® MES-SDS (NP0002). La corrida se realizó durante 50 minutos con una corriente inicial de $120 \mathrm{~mA} /$ gel y una tensión constante de $200 \mathrm{~V}$. Las bandas correspondientes a las subunidades individuales que conforman la estructura de la glicinina de soja y los hidrolizados se detectaron por tinción con plata (Blum et al., 1987) y para determinar el peso molecular se utilizó como marcador de peso molecular (Mark 12 Unstained standard).

\section{Actividad antioxidante}

La Capacidad de Absorción de Radicales de Oxígeno (ORACFL) se llevó a cabo de acuerdo con el método propuesto por Del Castillo et al. (2005), utilizando como antioxidante de referencia trolox.

La capacidad de atrapamiento de los radicales catiónicos se determinó usando el método de decoloración del radical ABTS descrito por Re et al. (1999), modificado por Plaza et al. (2010), en el que se utiliza como antioxidante de referencia trolox.

El Análisis de las Propiedades Antioxidantes de los hidrolizados Frente a Radicales hidroxilos se realizó siguiendo la técnica de degradación de la desoxirribosa, según el método propuesto por Halliwell et al. (1987), utilizando manitol como referencia.

Todas las determinaciones se realizaron al menos por triplicado.

El análisis estadístico de los datos se realizó mediante análisis de varianza (ANOVA) y test de mínima diferencia significativa (LSD) con un $(\mathrm{p} \leq 0.05)$ para comparación de las medias mediante el programa estadístico Statgraphics Plus 7.0.

\section{Resultados y Discusión}

Los resultados obtenidos por análisis de proteínas totales mediante el método de Kjeldhal indican un $(40.1 \pm 0.4) \%$ de proteína en los porotos de soja iniciales. Siguiendo el proceso de extracción se obtuvo a partir de éstos la fracción glicinina con $(95 \pm 1) \%$ de proteína total.

En la Figura 1 (electroforesis en gel de poliacrilamida en condiciones reductoras y desnaturalizantes) se observa las bandas correspondientes a las distintas subunidades de las proteínas de soja. En la glicinina los enlaces que estabilizan la estructura de la proteína se rompen por las condiciones del ensayo y permiten separar las subunidades ácidas con una masa molecular media de 36 kDa y las subunidades básicas con una masa molecular media de 22 $\mathrm{kDa}$ (calle 3). En la calle 2, correspondiente a la harina desgrasada obtenida a partir de los porotos de soja, se constata además de las 2 bandas correspondientes a la glicinina las bandas correspondientes a fracciones mayoritarias de la soja, como las subunidades $\alpha(63 \mathrm{kDa})$, $\alpha$ ' $(58 \mathrm{kDa})$ y $\beta(42 \mathrm{kDa})$ de la $\beta$-conglicinina (Bittencourt et al., 2005, 2007), proteína P34 (30 kDa) (Ogawa et al., 1991) y los inhibidores de proteasas de Kunitz (21 kDa) y de Bowman Birk (8 kDa) (Kato y Matsuda, 1997).

El termograma de glicinina obtenido por calorimetría diferencial de barrido presenta una endoterma alrededor de $92^{\circ} \mathrm{C}$ (Figura 2). Este resultado concuerda con Hermansson $(1978,1986)$ y Wagner y Añón (1990), quienes informan que en la proteína de soja se observan dos endotermas, una a temperatura de $95^{\circ} \mathrm{C}$ correspondiente a la glicinina y otra a temperaturas de $80{ }^{\circ} \mathrm{C}$, perteneciente a la $\beta$-conglicinina. Ambos resultados, la electroforesis y el DSC, reflejaron el alto grado de pureza presentado por el aislado de glicinina.

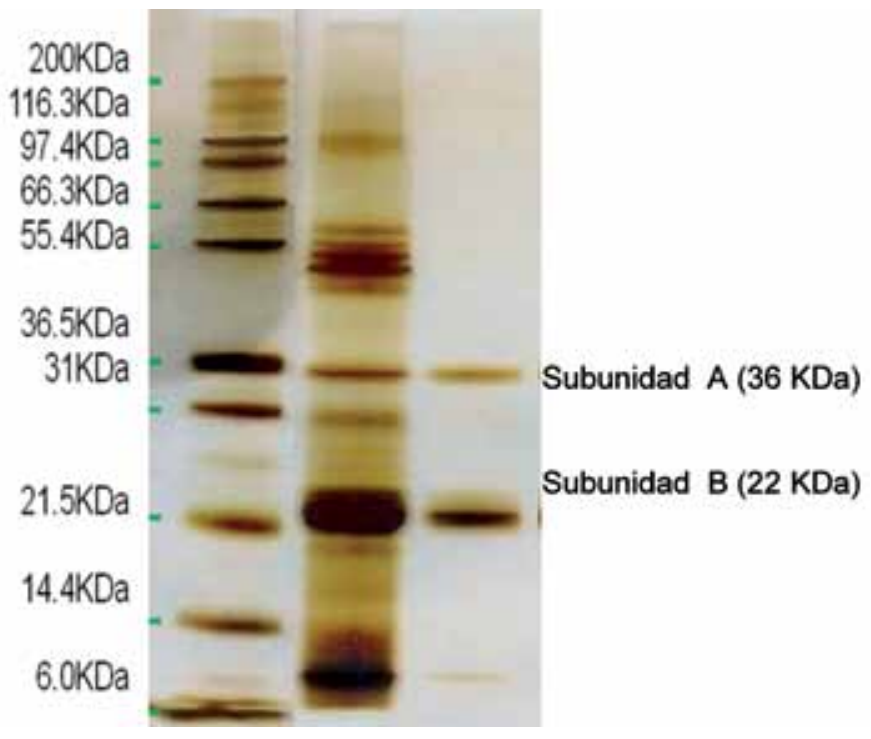

Figura 1. Electroforesis en geles de poliacrilamida SDS-PAGE (12\%) en condiciones reductoras y tinción de plata. Muestras: Marcador de peso molecular (calle 1), harina de soja desgrasada (calle 2), aislado de glicinina de soja (calle 3). 


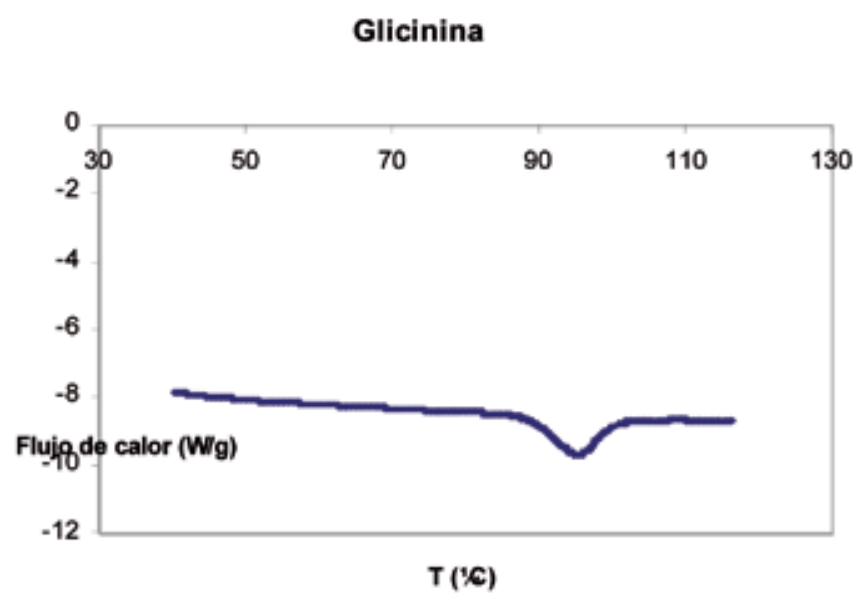

Figura 2. Termograma de Glicinina de soja aislada.

Una vez caracterizado el aislado se realizó la hidrólisis; los resultados indican que el grado de hidrólisis dependió del tiempo de reacción y de la naturaleza del preparado enzimático, con el resultado de que Flavourzyme es más activo que $\operatorname{PSG}(\mathrm{p} \leq 0.05)$ y alcanzó un grado de hidrólisis mayor al $50 \%$ a las 2 horas, mientras que con PSG se superó el $50 \%$ del GH a las 4 horas (Figura 3). Estos resultados correlacionaron con los presentados en la Figura 4 (electroforesis en geles de poliacrilamida), en el caso de la Flavourzyme calles 6 a 8 a las 2 horas de reacción (calle 7) desaparece la banda correspondiente a las subunidades ácida y básica de la glicinina y aparece un gran número de bandas con PM alrededor de 6Kda. Por otro lado, los hidrolizados obtenidos luego de 4 horas de reacción con PSG calle 4 presentaron $\mathrm{PM}$ menores a $14 \mathrm{KDa}$

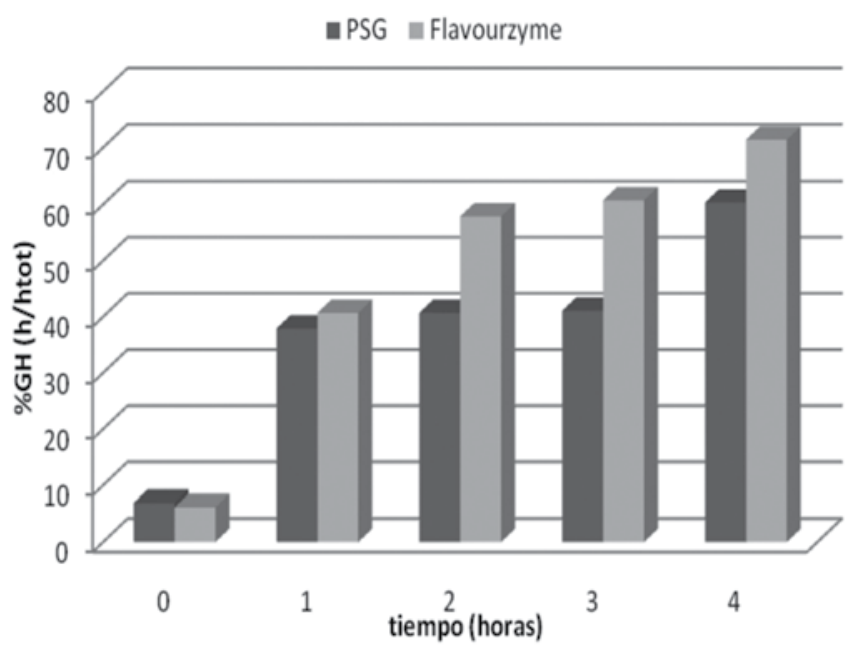

Figura 3. Grado de hidrólisis.

La acción de estas enzimas endo-exo peptidasas en las condiciones utilizadas resultaron ser eficientes para la obtención de péptidos con PM alrededor de $6 \mathrm{KDa}$. Estos resultados son similares a los descritos por otros autores para estas enzimas, con proteínas alimentarias tales como colágeno (Rosmus et al., 1967) y ovomucina (Hammershøj et al., 2008).

Los hidrolizados obtenidos con ambas enzimas presentaron propiedades antioxidantes frente a ABTS, ORAC y desoxirribosa.

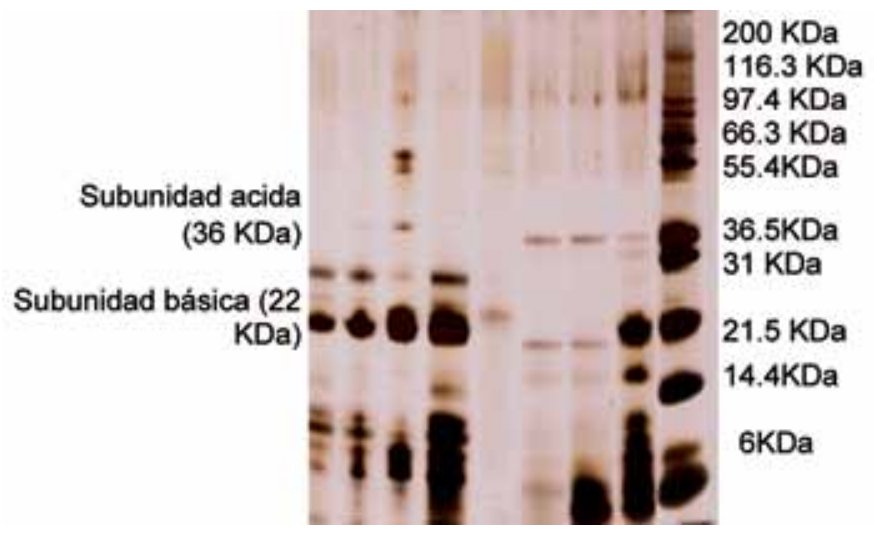

Figura 4. Electroforesis en geles de poliacrilamida SDS-PAGE (12\%) en condiciones reductoras y tinción de plata. Muestras hidrolizadas con PSG (calle 1), 1 hora ( (calle 2), 2 hora (calle 3), 3 hora (calle 4), 4 hora (calle 5), libre Hidrolizados con flavourzyme (calle 6), 3 hora (calle 7), 2 hora (calle 8), 1 hora patrón de PM (calle 9)

Los hidrolizados obtenidos a partir de Flavourzyme presentaron mayor capacidad de actuar frente a radicales ABTS e hidroxilos ( $\alpha$ $\leq 0.05$ ) que los hidrolizados con PSG (Figura 5). Estos resultados correlacionan con los descritos previamente por Moure et al. (2006), quienes encontraron valores de entre 820 y $3280 \mu$ moles TE/g de proteína para la actividad frente a los radicales ABTS; la fracción mas activa fue la de masa molecular inferior a los $10 \mathrm{kDa}$.

En cambio, los hidrolizados obtenidos por actividad de PSG presentaron mayor capacidad de actuar frente a radicales alquilperoxílicos $(\mathrm{p} \leq 0.05)$, como se ve en la Figura 5 . El grado de capacidad antioxidante parece estar relacionado con el grado de hidrólisis de las proteínas, a menor PM mayor actividad frente a radicales ABTS e hidróxilos.

Los compuestos fenólicos, como es el caso de isoflavonas (daidzeína y genisteína) presentes en la semilla de soja, son capaces de atrapar radicales libres (Arora et al., 1998; Setchell y Cole, 2003), presentando propiedades antioxidantes. Para confirmar que las propiedades antioxidantes encontradas se deben a los péptidos presentes y no a estos compuestos fenólicos -que podrían quedar durante el proceso de extracción de las proteínas- se realizó su determinación. Los resultados indicaron que no existen diferencias significativas $(p>0,05)$ para las muestras estudiadas, lo que sugiere que las diferencias en capacidad antioxidante de los hidrolizados pueden asociarse a los péptidos presentes. 

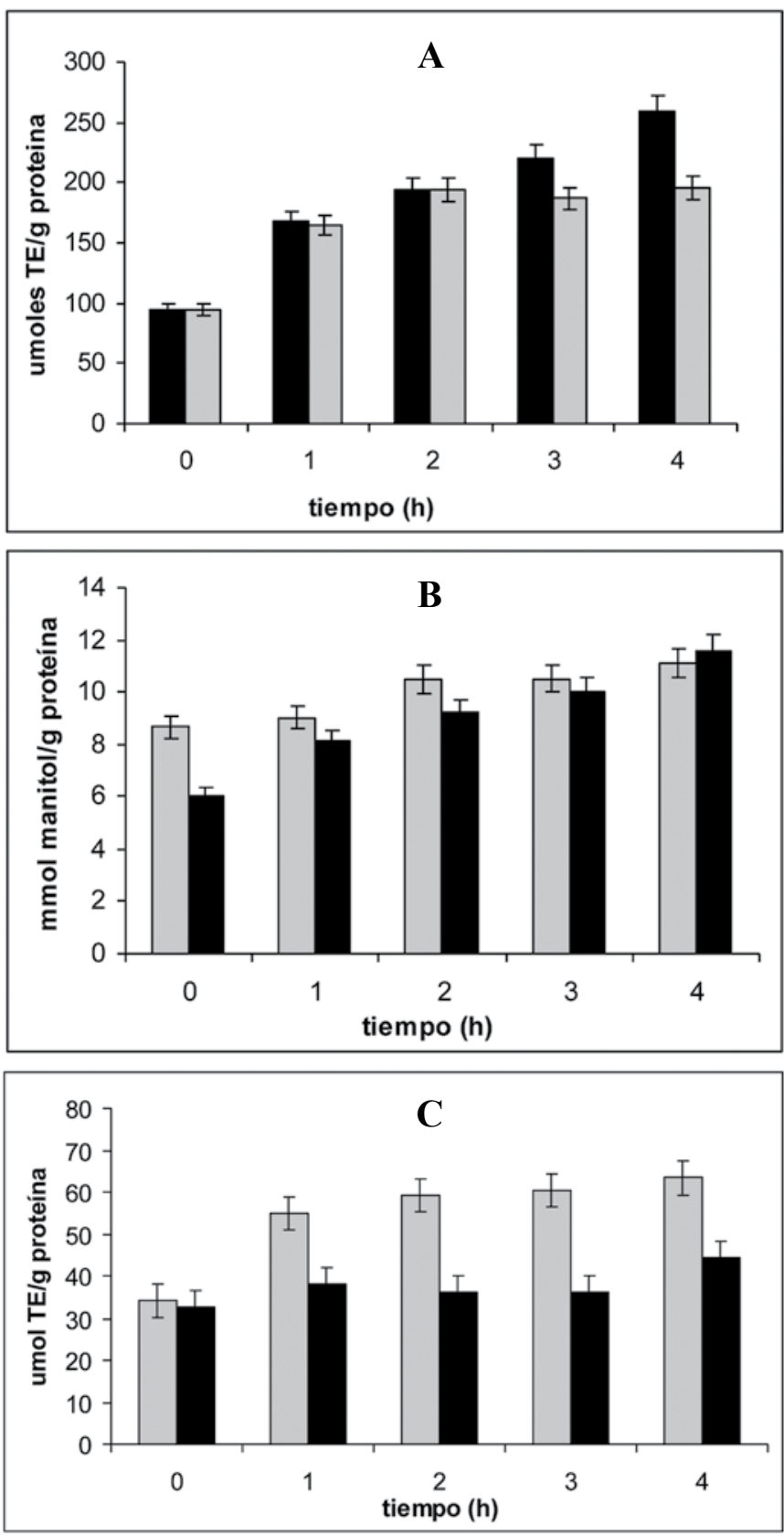

Figura 5. Determinación de la actividad antioxidante de los hidrolizados obtenidos por actividad de flavourzyme $\square$ y PSG a distintos tiempos de reacción por los métodos $(A)$ ABTS $(B)$ desoxirribosa (C) ORACFL.

\section{Conclusión}

Se concluyó que ambos preparados enzimáticos son efectivos para la obtención de péptidos antioxidantes de glicinina de soja. Resultan más efectivos frente a radicales ABTS e hidróxilos los hidrolizados de mayor $\% \mathrm{GH}$ obtenidos por acción de flavourzyme.

Dado que no existen diferencias significativas $(p>0,05)$ en las concentraciones de compuestos fenólicos de las muestras, las diferencias en capacidad antioxidante de los hidrolizados proteicos se puede asociar a su composición peptídica.

\section{Referencias}

- AOAC INTERNATIONAL. Official Methods of Analysis of AOAC International. 12a ed. Gaithersburg: AOAC, 1975. Official Method 14.026.

- AOAC INTERNATIONAL. Official Methods of Analysis of AOAC International. 15a ed. Gaithersburg: AOAC, 1990. Official Method 920.85 .
- ARORA, A.; NAIR, M.G.; STRASBURG, G.M. Antioxidant activities of isoflavones and their biological metabolites in liposomal system. En: Archives of Biochemistry and Biophysics 1998, 356(2):133-141

- BITTENCOURT, A.L.; FERNANDES, I.; PEREIRA, I.R.O.; HONMOTO, C.S.; TANAKA, M.K.; LIMA, F.S.P.; ABDALLA, D.S.P. Detection of $7 \mathrm{~S}$ and $11 \mathrm{~S}$ soy proteins fractions by monoclonal antibody-based immunoassays in food. En: Food and Agricultural Immunology 2005. 16(2):91-100.

- BITTENCOURT, A.L., SOARES, M.F., PIRES, R.R., HONMOTO, C.S., TANAKA, M.K., JACOB, C.M.; ABDALLA, D.S.P. Immunogenicity and allergenicity of $2 \mathrm{~S}, 7 \mathrm{~S}$ and $11 \mathrm{~S}$ soy proteins fractions. En: Brazilian Journal of Pharmaceutical Sciences. 2007, 43(4):597-606.

- BLUM, H.; BEIER, H.; GROSS, H.J. Improved silver staining of plant proteins, RNA and DNA polyacrilamide gels. En: Electrophoresis. 1987, 8(2):93-99.

- CHEN, H.M.; MURAMOTO, K.; YAMAUCHI, F. Structuralanalysis of antioxidative peptides from soybean beta-conglycinin. En: Journal of Agricultural and Food Chemistry. 1995, 43(3): 574-578.

- DEL CASTILLO, M.D.; GORDON, M.H.; AMES, J.M. Peroxyl radical-scavenging of coffe brews. En: European Food Research and Technology. 2005, 221(3-4):471-477.

- GÓMEZ, J.A.; LÓPEZ, I.; PIHLANTO, A.; RAMOS, M.; RECIO, I. Antioxidant activity of ovine casein hydrolysates: identification of active peptides by HPLC-MS/MS. En: European Food Research and Technology. 2008, 227(4):1061-1067.

- GOODNO, C.C.; SWAISGOOD, H.E.; CATIGNANI, G.L. A fluorimetric assay for available lysine in proteins. En: Analytical Biochemistry. 1981, 115(1):203-211.

- HALliWELL, B.; GUTTERIDGE, J.; ARUOMA, O. The Deoxyribose method: a simple "test-tube" assay for determination of rate constants for reactions of hydroxyl radicals. En: Analytical Biochemistry. 1987, 1652(15):2-19.

- HALLIWELL, B. Commentary oxidative stress, nutrition and health. Experimental strategies for optimization of nutritional antioxidant intake in humans. En: Free Radical Research. 1996a, 25(1):57-74.

- HALLIWELL, B. Antioxidants in human health and disease. En: Annual Review of Nutrition. 1996b, 16:33-50.

- HAMMERSHØJ, M.; NEBEL. C.; CARSTENS, J. H. Enzymatic hydrolysis of ovomucin and effect on foaming properties. En: Food Research International. 2008, 41(5):522-531.

- HERMANSSON, A. M. Physico-chemical aspects of soy proteins structure formation. En: Journal of Texture Studies. 1978, (9):3358.

- HERMANSSON, A. M. Soy protein gelation. En: Journal of the American Oil of Chemists 'Society. 1986, 63:658-666.

- HOGAN, S.; ZHANG, L.; LI, J.; WANG, H.; ZHOU, K. Development of antioxidant rich peptides from milk protein by microbial proteases and analysis of their effects on lipid peroxidation in cooked beef. En: Food Chemistry. 2009, 117(3):438-443.

- KATO, Y.; MATSUDA, T. Glycation of proteinous inhibitors: loss in trypsin inhibitory activity by blocking of arginine and lysine residues at their reactive sites. En: Journal of Agricultural and Food Chemistry. 1997, 45(10):3826-3831.

- KOSAR, M.; DORMAN, H.J.; HILTUNEN, R. Effect of an acid treatment on the phytochemical and antioxidant characteristics of extracts from selected Lamiaceae species. En: Food Chemistry. 2005, 91:525-533.

- LIU, H.C.; CHEN, W.L.; MAO, S.J.T. Antioxidant nature of bovine milk $\beta$-Lactoglobulin. En: Journal of Dairy Science. 2007, 90(2):547-555.

- LOWRY, O.; ROSEBROUGH, N.; FARR, A.; RANDALL, R. Protein measurement with the folin-phenol reagent. En: Journal of Biological Chemistry. 1951, 193:265-275.

- MOURE, A.; DOMÍNGUEZ, H.; PARAJÓ, J.C. Antioxidant 
properties of ultrafiltration-recovered soy protein fractions from industrial effluents and their hydrolysates. En: Process Biochemistry. 2006, 41 (2):447-456.

- NAGANO, T.; HIROTSUKA, M.; HIROYUKI M.; KOHYAMA, K.; Y NISHINARI K. Dynamic viscoelastic study on the gelation of 7S Globulin from soybeans. En: Food Chemistry. 1992, 40:941944.

- NIELSEN, N.C. The structure and complexity of the 11S polypeptides in soybeans. En: Journal of the American Oil Chemists Society. 1985, 62(12):1680-1686.

- OGAWA, T.; BANDON, N.; TSUJI, H.; OKAJIMA, H.; NISHIKAWA, K.; SASAOKA, K. Investigation of the IgE-binding proteins in soybeans by immunoblotting with sera of the soybeansensitive patients with atopic-dermatitis. En: Journal of Nutritional Science and Vitaminology. 1991, 37(6):555-565.

- PLAZA, M.; AMIGO M.; DEL CASTILLO, M.D.; IBÁÑEZ, E.; HERRERO, M. Facts about the formation of new antioxidants in natural samples after subcritical water extraction. En: Food Research International. 2010, 43:2341-2348.

- RE, R.; PELLEGRINI, N.; PROTEGGENTE, A.; PANNALA, A.; YANG, M.; RICE-EVANS, C. Antioxidant activity applying an improved ABTS radical catión decoloration assay. En: Free Radical Biology and Medicine. 1999, 26(9-10):1231-1237.

- ROSMUS, J.; DEYL, Z.; DRAKE, M.P. Studies on the structure of collagen: I. The sequence analysis of peptides released by pronase. En: Biochimica et Biophysica Acta - Protein Structure. 1967, 140(3):507-514.

- SAKANAKA, S.; TACHIBANA, Y.; ISHIHARA, N.; JUNEJA, L.R. Antioxidant activity of egg-yolk protein hydrolyzates in linoleic acid oxidation system. En: Food Chemistry. 2004, 86(1):99-103.

- SAKANAKA, S.; TACHIBANA, Y. Active oxygen scavenging activity of egg-yolk protein hydrolyzates and their effects on lipid oxidation in beef and tuna homogenates. En: Food Chemistry. 2006, 95(2): 243-249.

- SETCHELL, K.D.R.; COLE, S.J. Variations in isoflavone levels in soy foods and soy protein isolates and issues related to isoflavone databases and food labelling. En: Journal of Agricultural and Food Chemistry. 2003, 51(14):4146-4155.

- STASWICK, P.E.; HERMODSON, M.A.; NIELSEN, N.C. Identification of the cystines which link the acidic and basic components of the glycinin subunits. En: The Journal of Biological Chemistry. 1984, 259(21):13431-13435.

- STATPOINT TECHNOLOGIES, INC. Statgraphics Plus [Software]. 7.0. Warrenton: Statpoint Technologies, INC., [s.d.].

- UCHIDA, K.; KAWAKISHI, S. Sequence-dependent reactivity of histidine-containing peptides with copper(II)/ ascorbate. En: Journal of Agricultural Food Chemistry. 1992, 40:13-16.

- WAGNER, J. R.; AÑÓN, M. C. Influence of denaturation, hydrophobicity and sulfhidryl content on solubility and water absorbing capacity of soy protein isolate. En: Journal of Food Science. 1990, 50:765-770. 